\title{
Measurement of $\mathrm{SO}_{2}$ Solubility in Ionic Liquids
}

Jessica L. Anderson, JaNeille K. Dixon, Edward J. Maginn*, Joan F. Brennecke*

Department of Chemical and Biomolecular Engineering University of Notre Dame, Notre Dame, IN 46556 U.S.A.

* denotes corresponding authors, ed@nd.edu, jefb@nd.edu

\section{Supporting Information}

The materials used in this study, including source, grade, and purification method (if any), are as follows: sodium bromide (Aldrich $\geq 99.0 \%$, used as received), 1-chlorobutane (Aldrich 99.5\%, redistilled), 1-bromohexane (Aldrich $\geq 98.0 \%$, redistilled), sodium dicyanoamide (Aldrich 96\%, used as received), pyridine (Aldrich $\geq 99.0 \%$, redistilled over $\mathrm{KOH}$ ), 3,5-dimethylpyridine (Aldrich $\geq 99.0 \%$, redistilled over $\mathrm{KOH}$ ), 4-dimethylaminopyridine (Reilly 99\%, used as received), 1-methylimidazole (Aldrich 99\%, redistilled over $\mathrm{KOH}$ ), and lithium bis(trifluoromethanesulfonyl)imide (3 M 97\%, used as received).

1-hexyl-3-methylimidazolium bis(trifluoromethylsulfonyl)imide: ${ }^{1} \mathrm{H} \mathrm{NMR}$ (cation, $\mathrm{CDCl}_{3}$ ): $\delta=8.99(\mathrm{~s}, 1), \delta=7.40(\mathrm{t}, J=1.8 \mathrm{~Hz}, 1), \delta=7.36(\mathrm{t}, J=1.8 \mathrm{~Hz}, 1), \delta=4.13(\mathrm{t}, J=7.5 \mathrm{~Hz}, 2), \delta$

$=3.91(\mathrm{~s}, 3), \delta=1.81(\mathrm{~m}, J=6.9 \mathrm{~Hz}, 2), \delta=1.25(\mathrm{~m}, 6), \delta=0.81(\mathrm{t}, J=6.9 \mathrm{~Hz}, 3) \cdot{ }^{13} \mathrm{C} \mathrm{NMR}$ (cation, $\left.\mathrm{CDCl}_{3}\right): \delta=136.43(\mathrm{CH}), \delta=123.85(\mathrm{CH}), \delta=122.344(\mathrm{CH}), \delta=50.06\left(\mathrm{CH}_{2}\right), \delta=$ $36.28\left(\mathrm{CH}_{2}\right), \delta=30.99\left(\mathrm{CH}_{3}\right), \delta=30.01\left(\mathrm{CH}_{2}\right), \delta=25.75\left(\mathrm{CH}_{2}\right), \delta=22.31\left(\mathrm{CH}_{2}\right), \delta=13.86$ $\left(\mathrm{CH}_{3}\right) \cdot{ }^{13} \mathrm{C}$ NMR (anion, $\left.\mathrm{CDCl}_{3}\right): \delta=121\left(\mathrm{q}, J=322 \mathrm{~Hz}, \mathrm{CF}_{3}\right)$.

1-hexyl-3-methylpyridinium bis(trifluoromethylsulfonyl)imide: ${ }^{1} \mathrm{H}$ NMR $\left(\mathrm{CDCl}_{3}\right): \delta=8.82$ $(\mathrm{s}, 1), \delta=8.75(\mathrm{~d}, J=5.7 \mathrm{~Hz}, 1), \delta=8.44(\mathrm{~d}, J=7.8 \mathrm{~Hz}, 1), \delta=8.01(\mathrm{t}, J=5.7 \mathrm{~Hz}, 1), \delta=4.63$ $(\mathrm{t}, J=7.5 \mathrm{~Hz}, 2), \delta=2.61(\mathrm{~s}, 3), \delta=2.04(\mathrm{~m}, 2), \delta=1.32(\mathrm{~m}, 6), \delta=0.84(\mathrm{t}, J=6.9 \mathrm{~Hz}, 3) .{ }^{13} \mathrm{C}$ $\operatorname{NMR}\left(\mathrm{CDCl}_{3}\right): \delta=146.28(\mathrm{CH}), \delta=143.99(\mathrm{CH}), \delta=141.61(\mathrm{CH}), \delta=140.13(\mathrm{CH}), \delta=$ 
$146.28(\mathrm{CH}), \delta=127.76(\mathrm{C}), \delta=61.99\left(\mathrm{CH}_{2}\right), \delta=30.87\left(\mathrm{CH}_{2}\right), \delta=30.67\left(\mathrm{CH}_{3}\right), \delta=25.20$

$\left(\mathrm{CH}_{2}\right), \delta=22.06\left(\mathrm{CH}_{2}\right), \delta=18.12\left(\mathrm{CH}_{2}\right), \delta=13.65\left(\mathrm{CH}_{3}\right) .{ }^{13} \mathrm{C} \mathrm{NMR}\left(\right.$ anion, $\left.\mathrm{CDCl}_{3}\right): \delta=121$

$\left(\mathrm{q}, J=322 \mathrm{~Hz}, \mathrm{CF}_{3}\right)$.

Solubility Tables

Table S1: $\mathrm{SO}_{2}$ solubility in $[\mathrm{hmim}]\left[\mathrm{Tf}_{2} \mathrm{~N}\right]$

\begin{tabular}{|c|c|c|c|c|c|c|c|c|}
\hline \multicolumn{3}{|c|}{$2{ }^{\circ} \mathrm{C}$} & \multicolumn{3}{c|}{$40{ }^{\circ} \mathrm{C}$} & \multicolumn{3}{c|}{$60{ }^{\circ} \mathrm{C}$} \\
\hline $\begin{array}{c}\text { Fugacity } \\
\text { (bar) }\end{array}$ & $\begin{array}{c}\text { Mole } \\
\text { Fraction } \\
\mathrm{SO}_{2}\end{array}$ & $\begin{array}{c}\text { Mole } \\
\text { Fraction } \\
\text { Uncertainty }\end{array}$ & $\begin{array}{c}\text { Fugacity } \\
\text { (bar) }\end{array}$ & $\begin{array}{c}\text { Mole } \\
\text { Fraction } \\
\text { SO }\end{array}$ & $\begin{array}{c}\text { Mole } \\
\text { Fraction } \\
\text { Uncertainty }\end{array}$ & $\begin{array}{c}\text { Mole } \\
\text { Fugacity } \\
\text { (bar) }\end{array}$ & $\begin{array}{c}\text { Mole } \\
\text { Fraction } \\
\text { SO }{ }_{2}\end{array}$ & $\begin{array}{c}\text { Fraction } \\
\text { Uncertainty }\end{array}$ \\
\hline $5.94 \mathrm{E}-01$ & $3.10 \mathrm{E}-01$ & $2.51 \mathrm{E}-03$ & $2.99 \mathrm{E}-01$ & $1.03 \mathrm{E}-01$ & $2.53 \mathrm{E}-03$ & $3.29 \mathrm{E}-01$ & $6.76 \mathrm{E}-02$ & $2.57 \mathrm{E}-03$ \\
\hline $9.94 \mathrm{E}-01$ & $4.78 \mathrm{E}-01$ & $2.51 \mathrm{E}-03$ & $5.76 \mathrm{E}-01$ & $2.28 \mathrm{E}-01$ & $2.53 \mathrm{E}-03$ & $5.76 \mathrm{E}-01$ & $1.36 \mathrm{E}-01$ & $2.57 \mathrm{E}-03$ \\
\hline $1.49 \mathrm{E}+00$ & $6.16 \mathrm{E}-01$ & $2.51 \mathrm{E}-03$ & $9.77 \mathrm{E}-01$ & $3.67 \mathrm{E}-01$ & $2.53 \mathrm{E}-03$ & $1.04 \mathrm{E}+00$ & $2.48 \mathrm{E}-01$ & $2.57 \mathrm{E}-03$ \\
\hline $1.98 \mathrm{E}+00$ & $7.11 \mathrm{E}-01$ & $2.51 \mathrm{E}-03$ & $1.69 \mathrm{E}+00$ & $5.33 \mathrm{E}-01$ & $2.53 \mathrm{E}-03$ & $1.71 \mathrm{E}+00$ & $3.74 \mathrm{E}-01$ & $2.57 \mathrm{E}-03$ \\
\hline $2.45 \mathrm{E}+00$ & $7.81 \mathrm{E}-01$ & $2.51 \mathrm{E}-03$ & $2.47 \mathrm{E}+00$ & $6.55 \mathrm{E}-01$ & $2.53 \mathrm{E}-03$ & $2.45 \mathrm{E}+00$ & $4.88 \mathrm{E}-01$ & $2.57 \mathrm{E}-03$ \\
\hline $2.94 \mathrm{E}+00$ & $8.44 \mathrm{E}-01$ & $2.51 \mathrm{E}-03$ & $2.97 \mathrm{E}+00$ & $7.19 \mathrm{E}-01$ & $2.53 \mathrm{E}-03$ & $3.19 \mathrm{E}+00$ & $5.80 \mathrm{E}-01$ & $2.57 \mathrm{E}-03$ \\
\hline $2.37 \mathrm{E}+00$ & $7.82 \mathrm{E}-01$ & $2.51 \mathrm{E}-03$ & $2.58 \mathrm{E}+00$ & $6.71 \mathrm{E}-01$ & $2.53 \mathrm{E}-03$ & $2.42 \mathrm{E}+00$ & $4.84 \mathrm{E}-01$ & $2.57 \mathrm{E}-03$ \\
\hline $1.43 \mathrm{E}+00$ & $6.16 \mathrm{E}-01$ & $2.51 \mathrm{E}-03$ & $2.11 \mathrm{E}+00$ & $6.04 \mathrm{E}-01$ & $2.53 \mathrm{E}-03$ & $1.61 \mathrm{E}+00$ & $3.57 \mathrm{E}-01$ & $2.57 \mathrm{E}-03$ \\
\hline $4.47 \mathrm{E}-01$ & $2.49 \mathrm{E}-01$ & $2.51 \mathrm{E}-03$ & $1.24 \mathrm{E}+00$ & $4.36 \mathrm{E}-01$ & $2.53 \mathrm{E}-03$ & $7.98 \mathrm{E}-01$ & $1.94 \mathrm{E}-01$ & $2.57 \mathrm{E}-03$ \\
\hline $2.39 \mathrm{E}-01$ & $9.93 \mathrm{E}-02$ & $2.51 \mathrm{E}-03$ & $4.37 \mathrm{E}-01$ & $1.65 \mathrm{E}-01$ & $2.53 \mathrm{E}-03$ & $2.79 \mathrm{E}-01$ & $4.91 \mathrm{E}-02$ & $2.57 \mathrm{E}-03$ \\
\hline
\end{tabular}

Table S2: $\mathrm{SO}_{2}$ solubility in [hmpy][ $\left.\mathrm{Tf}_{2} \mathrm{~N}\right]$

\begin{tabular}{|c|c|c|}
\hline \multicolumn{3}{|c|}{$2{ }^{\circ} \mathrm{C}$} \\
\hline $\begin{array}{c}\text { Fugacity } \\
\text { (bar) }\end{array}$ & $\begin{array}{c}\text { Mole } \\
\text { Fraction } \\
\mathrm{SO}_{2}\end{array}$ & $\begin{array}{c}\text { Mole } \\
\text { Fraction } \\
\text { Uncertainty }\end{array}$ \\
\hline $2.89 \mathrm{E}-01$ & $1.38 \mathrm{E}-01$ & $3.38 \mathrm{E}-03$ \\
\hline $6.17 \mathrm{E}-01$ & $3.43 \mathrm{E}-01$ & $3.38 \mathrm{E}-03$ \\
\hline $1.10 \mathrm{E}+00$ & $5.22 \mathrm{E}-01$ & $3.38 \mathrm{E}-03$ \\
\hline $1.67 \mathrm{E}+00$ & $6.60 \mathrm{E}-01$ & $3.38 \mathrm{E}-03$ \\
\hline $2.37 \mathrm{E}+00$ & $7.75 \mathrm{E}-01$ & $3.38 \mathrm{E}-03$ \\
\hline $2.96 \mathrm{E}+00$ & $8.54 \mathrm{E}-01$ & $3.38 \mathrm{E}-03$ \\
\hline $2.65 \mathrm{E}+00$ & $8.15 \mathrm{E}-01$ & $3.38 \mathrm{E}-03$ \\
\hline $2.21 \mathrm{E}+00$ & $7.54 \mathrm{E}-01$ & $3.38 \mathrm{E}-03$ \\
\hline $1.56 \mathrm{E}+00$ & $6.40 \mathrm{E}-01$ & $3.38 \mathrm{E}-03$ \\
\hline $7.90 \mathrm{E}-01$ & $4.21 \mathrm{E}-01$ & $3.38 \mathrm{E}-03$ \\
\hline $4.47 \mathrm{E}-01$ & $2.45 \mathrm{E}-01$ & $3.38 \mathrm{E}-03$ \\
\hline
\end{tabular}

